\title{
Development of Integral Smart Home Appliances
}

\author{
Fengzhi Dai ${ }^{1 *}$, Yuxing Ouyang ${ }^{1}$, Yiqiao Qin ${ }^{1}$, Ce Bian ${ }^{1}$, Baochang Wei ${ }^{1}$, Shengbiao Chang ${ }^{1}$, Bo Liu ${ }^{2}$ \\ ${ }^{1}$ College of Electronic Information and Automation, Tianjin University of Science and Technology, \\ 1038 Dagunanlu Road, Hexi District, Tianjin, 300222, China \\ E-mail: ${ }^{*}$ daifz@tust.edu.cn \\ www.tust.edu.cn \\ ${ }^{2}$ Inner Mongolia University, China
}

\begin{abstract}
The integral smart home appliances are developed for monitoring and control the household devices. The WI-FI module is used for wireless network communication, so as to upload information of all connected smart home appliances to the server. The home appliances can also be remotely controlled by the external network. The Airkiss distribution mode is used to connect the WI-FI master module to the network, and to connect to other WI-FI module devices to adopt the ad hoc network mode for wireless networking.
\end{abstract}

Keywords: smart home appliance, remote control and monitoring, WI-FI, ad hoc network, Airkiss network

\section{Introduction}

With the rapid development of the Internet of Things (IoT) ${ }^{1}$, many of the traditional home appliances have gradually been developed into smart home devices ${ }^{2}$, such as the smart sockets, smart clock, smart closestool, etc.

Compared to the traditional home appliances, the smart home appliances have the function of monitoring and control. Since each device needs to be controlled in a different way, it is very difficult to realize the integrated control of all kinds of home appliances together by using the network remote control.

To solve this problem, the design focuses on the integration of smart home appliances. The purpose of the design is to create a comfortable, convenient and fast intelligent home living environment ${ }^{3}$, so that our smart home life can be further optimized and improved.

The wireless communication is used to control the different devices, and to upload the collected data from these devices to the server and receive command from the server. The system structure is shown in Fig.1.

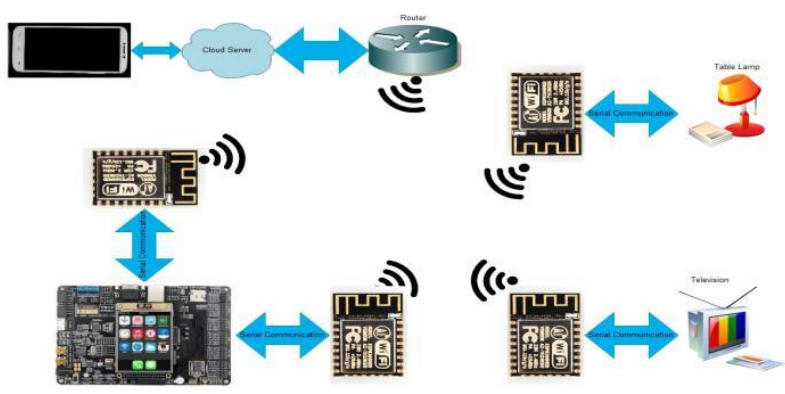

Fig.1 The system structural block diagram

Just as shown in Fig.1, this design adopts the wireless ad hoc network mode of WI-FI module. So that the local WI-FI module can set up the local LAN network, and achieve the purpose of connecting all the smart home appliances. The WI-FI master module connects to the server through the Airkiss protocol, which can control and monitor the intelligent household devices. 
In this paper, Section 2 describes the hardware circuit design, and Section 3 introduces the function of some protocols. The experimental results are given in Section 4 , and the conclusions are presented in Section 5.

\section{The hardware circuit design}

For the hardware circuit design, we use Altium Designer ${ }^{4}$ to draw the schematic. The hardware circuit is divided into the terminal circuit and equipment circuit. The terminal circuit is discussed in Section 2-1, and Section 2-2 describes the equipment circuit.

\section{2-1. The terminal circuit}

The terminal circuit uses the STM32 chip ${ }^{5}$ as the master chip, which is the Cortex-M3 ARM core and has 144 pins. The chip has 512KB flash memory and 64KB RAM $^{6}$. It can download and online debug the program through SWD or JTAG with KEIL 4, and has the function of one-key-download. It has more than 80 general-purpose IO ports, multiple timers and serial ports. The STM32 minimum system circuit diagram is shown in Fig.2.

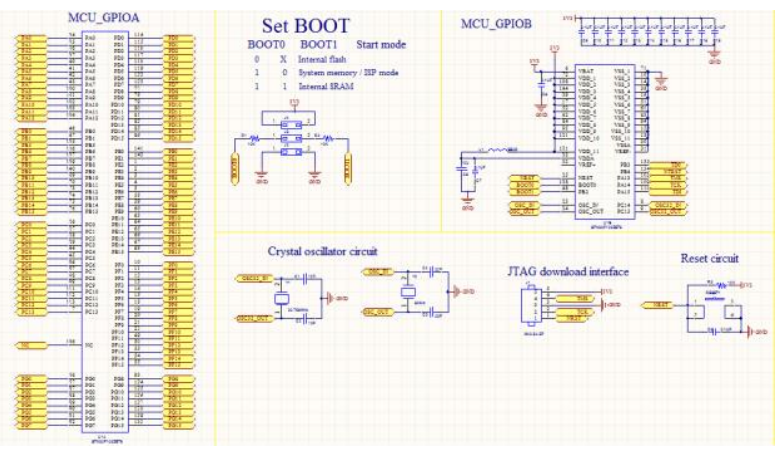

Fig.2 STM32 minimum system circuit diagram

\section{2-2. The equipment circuit}

The equipment circuit uses the ESP8266 WI-FI module ${ }^{6}$ to realize the function of transmitting data to the terminal equipment and receiving data from the terminal equipment. The control of the device is also realized by the equipment circuit. The WI-FI module has the following characteristics:

(1) A built-in 10-bit high-precision ADC, with a complete TCP / IP protocol stack.
(2) Supports Cloud Server Development / Firmware and SDK for fast on-chip programming.

(3) Wide temperature range: $-40{ }^{\circ} \mathrm{C} \sim 125^{\circ} \mathrm{C}$.

(4) Operating Voltage: $3.0 \mathrm{~V} \sim 3.6 \mathrm{~V}$.

The minimum system circuit diagram of the WI-FI module is shown in Fig.3.

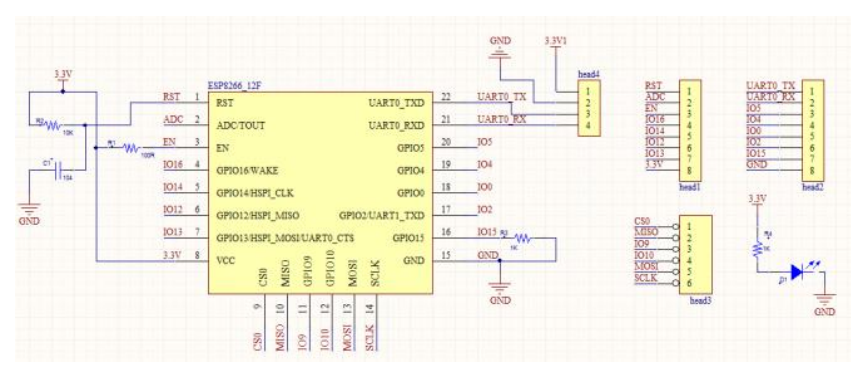

Fig.3. WI-FI module minimum system circuit diagram

\section{The function of some protocols}

A variety of protocols are used to make the function more perfect. We use ESP8266 built-in TCP/IP protocol for device network connectivity. And the MESH protocol is used for the group network, so that the equipment can be communicated each other. Also we use the Airkiss protocol to configure the network, which can greatly improve the convenience of the configuration.

Section 3-1 describes the MESH protocol, and Section 3-2 introduces the Airkiss protocol.

\section{3-1 Mesh protocols}

The design uses a wireless Mesh network ${ }^{7}$ for the ad hoc network communication. In the mesh network structure, the number can be determined by the number of nodes.

The relationship between the maximum mount numbers $(\mathrm{L})$ and the maximum hop numbers $(\mathrm{N})$ can be expressed as:

$$
L=4^{0}+4^{1}+\ldots+4^{N-1}
$$

The system uses the automatic network mode: As soon as the firmware is downloaded to the module, the WI-FI master module will connect to the router, and then the router is connected to the WAN. Other sub-modules will automatically scan around the WI-FI access points (AP). The WI-FI master module can receive, transmit and forward data packets, while the 
other sub-modules can only receive or send data packets. The ESP8266 MESH network is shown in Fig.4.

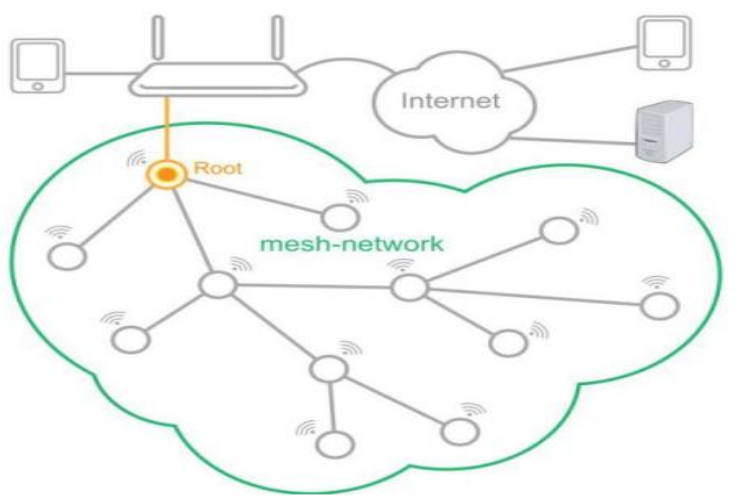

Fig.4 ESP8266 MESH network

\section{3-2 Airkiss protocols}

With the mobile-phone communicational APP WeChat ${ }^{8}$, the WI-FI module of the system can be carried out by Airkiss distribution network, which makes WI-FI module access to the Internet.

The principle of the Airkiss distribution network is that, through the discovery and promiscuous mode of LAN to get the smart configuration. Since the SDK programming and the firmware is burned to the WI-FI module, the WI-FI module can access to the network through the phone.

The steps of Airkiss smart configuration are as follows:

(1) Scan the two-dimensional (QR) code by WeChat.

(2) Fill the SSID and password in the pop-up window.

(3) WI-FI master module receives the SSID and password, and automatically connects to the router.

\section{Experimental results}

Over ten thousands of times, the data transmission experiments have been done. Section 4-1 shows the experimental results for the different communication distances between the terminal device and the sub-device. Section 4-2 shows the experimental results for the maximum number of hops for mesh networks. A simplified version of the terminal device and the sub-device is shown in Fig.5.

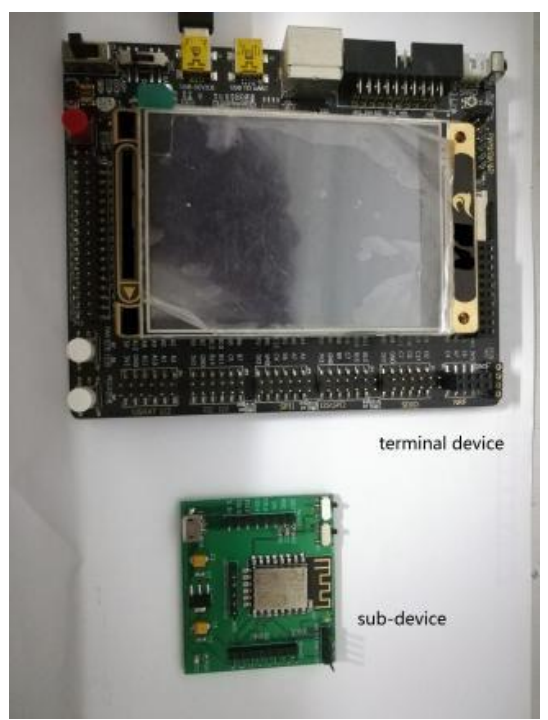

Fig.5 terminal device and sub-device

\section{4-1 Experiment of communication distance}

In the case of setting up obstacles and without obstacles, we test the different communication distances between the terminal equipment and the sub-devices.

For each experiment of different communication distances, we have carried out ten thousands data transmission. The results of setting and without setting obstacles are shown in Tab. 1 and Tab. 2 respectively.

Tab.1 Communication distance - setting obstacle

\begin{tabular}{cccc}
\hline & 20 meters & 30 meters & 40 meters \\
\hline $\begin{array}{c}\text { Number of } \\
\text { experimental } \\
\text { times }\end{array}$ & 11729 & 10249 & 15015 \\
\hline Success rate & $100 \%$ & $99.84 \%$ & $93.05 \%$ \\
\hline
\end{tabular}

Tab.2 Communication distance - without obstacle

\begin{tabular}{cccc}
\hline & 30 meters & 48 meters & 90 meters \\
\hline $\begin{array}{c}\text { Number of } \\
\text { experimental } \\
\text { times }\end{array}$ & 10183 & 10028 & 10611 \\
\hline Success rate & $100 \%$ & $100 \%$ & $99.29 \%$ \\
\hline
\end{tabular}

In case of setting obstacles: within 20 meters, no loss of data. For 30 meters distance, the data appears in a 
small amount of packet loss. And for 40 meters, more the data are lost.

In case of no obstacle: within 48 meters there is no data loss, and the data appears has a small amount of packet loss for experiment with 90 meters distance between the terminal equipment and the sub-devices.

\section{4-2 Maximum hops test of mesh network}

Refered to the official data sheet, we finally set the mesh network maximum hop count to 4 jumps. Without setting the obstacles, the result is shown in Tab.3.

Tab.3 Communication distance - without obstacle

\begin{tabular}{cccc}
\hline Number of hops & 2 & 3 & 4 \\
\hline Distance (m) & 30 & 60 & 90 \\
\hline Success rate (\%) & 100 & 100 & 100 \\
\hline
\end{tabular}

\section{Conclusion}

By the test of the smart home devices, the following data are obtained: The Mesh network allows up to 4 hops, and each mesh-non-leaf node can allow up to 4 direct child nodes to access the network with $100 \%$ success rate. When the transmission distance is within 20 meters, there is no packet loss, and the router can mount a Mesh network with a network size of 80 nodes.

The above data shows that the device can basically meet the needs of smart home appliances integration.

\section{Acknowledgements}

The research is partly supported by the Research Fund for the Reform in Education from Tianjin Municipal Education Commission of China (171005704B), and the key technologies $\mathrm{R} \& \mathrm{D}$ program of Tianjin (14ZCZDSY00010). It is also supported by the Science $\&$ Technology Fund Planning Project of Tianjin Higher Schools (20120831, 20140710-1400020005).

\section{References}

1. Kuen-Min Lee, Wei-Guang Teng, Ting-Wei Hou. Point-n-Press: An Intelligent Universal Remote Control System for Home Appliances. IEEE transactions on automation science and engineering, 13(3): 1308-1317, 2016.

2. N. Bitterman, D. Shach-Pinsly. Smart home-a challenge for architects and designers. Architectural science review, 58(3): 266-274, 2015.

3. Ju-Jang Lee, Kap-Ho Seo, Changmok Oh, Z. Zenn Bien. Development of a future Intelligent Sweet Home for the disabled. Artificial life and robotics, 11(1): 8-12, 2007.

4. Alexander S. Nikitin, Fedor G. Zograf, Alexander M. Fen, Sergey I. Tregubov. Specials of Electrical Wiring in Altium Designer \& SolidWorks. 2013 International Siberian Conference on Control and Communications, 2013.

5. Yuanxin Lin, Rui Kong, Rongbin She, Shugao Deng. Design and Implementation of Remote/Short-range Smart Home Monitoring System Based on ZigBee and STM32. Research journal of applied science, engineering and technology, 5(9): 2792-2798, 2013.

6. Qazi Mamoon Ashraf, Mohd Izhan Mohd Yusoff, Amir Alif Azman, Norbaizura Mohd Nor, Nor Aliya Ahmad Fuzi, Mohd Shahril Saharedan, Nurul Afzan Omar. Energy Monitoring Prototype for Internet of Things: Preliminary Results. 2015 IEEE 2nd World Forum on Internet of Things, 2015.

7. Andres Arjona, Cedric Westphal, Jukka Manner, Antti Yla-Jaaski, Sami Takala. Can the current generation of wireless mesh networks compete with cellular voice? Computer communications, 31(8): 1564-1578, 2008.

8. Chunmei Gan. A survey of WeChat application in Chinese public libraries. Library hi tech, 34(4): 625-638, 2016. 